\title{
4. Resümee: die Logik der Standardisierung und Vorteile der Technischen Regulierung
}

Standardisierung ist ein natürlicher Prozess in der Entwicklung einer Gesellschaft, der unabhängig von der Produkt- oder Prozessart auf allen Ebenen wirtschaftlicher und anderer gesellschaftlicher Beziehungen und in allen Ländern und Gemeinschaften stattfindet. Die dargestellten Parallelen in der Entwicklung der Standards, der Zollunionen und der Technischen Regulierung in Russland und in der Welt und die Rollen diverser Institutionen auf dem Gebiet der Standardisierung verdeutlichen die Bedeutung, den Sinn und die Gemeinsamkeiten solcher Prozesse und schaffen das nötige Verständnis für auf den ersten Blick unverständliche und bürokratische Prozeduren bei der Realisierung von Investitions-projekten in Russland.

Die Fülle an Bestimmungen und Vorschriften lässt sich in eine strukturierte Ordnung bringen, indem deren Hierarchien systematisch aufgezeigt und die Rolle der jeweiligen Institutionen in den Prozessen explizit dargestellt werden. Dieser Prozess des „Ordnung-Schaffens“ ist nichts anderes als die Standardisierung selbst. Als Prozess der Optimierung und der Systematisierung der normativ-rechtlichen Dokumente kann die Standardisierung als eine fortlaufende kontinuierliche Aufgabe aller Beteiligten gesehen werden.

Die Reform der Technischen Regulierung in der Eurasischen Wirtschaftsunion schreitet zwar langsamer als erhofft, doch kontinuierlich und bringt Vorteile nicht nur für die Mitglieder der Gemeinschaft, sondern auch für die „Außenstehenden“, indem die Regulierungsprozesse der Mitgliedsländer vereinheitlicht werden. Dadurch verringert sich die Zahl der notwendigen Regulierungsprozeduren, denen Exporteure immer ausgesetzt sind. 